\title{
Design of a Co-Planar Airborne Separation Display
}

\author{
Joost Ellerbroek, Student member, IEEE, Koen C. R. Brantegem, M. M. (René) van Paassen, Member, IEEE, \\ Max Mulder
}

\begin{abstract}
This paper describes a concept for a co-planar airborne self-separation display, that is designed to aid pilots in their separation task, by visualizing the possibilities for conflict resolution that the airspace provides. This work is part of an ongoing research towards the design of a constraint-based 3-D separation assistance interface that can present all the relevant properties of the spatio-temporal separation problem. A display concept is proposed that presents speed, heading and altitude action possibilities in two planar projections of the maneuver action-space. The interface also visualizes how these projections interact with each other.
\end{abstract}

Index Terms-Ecological Interface Design (EID), Airborne Separation Assistance System (ASAS), self-separation, situation awareness

\section{INTRODUCTION}

$\mathbf{I}$ $\mathrm{N}$ THE CURRENT Air-Traffic Management (ATM) concepts for unmanaged airspace, aircraft will fly optimized, four-dimensional trajectories, that have been determined and coordinated completely before the actual flight [1], [2]. To resolve traffic (or other) conflicts that result from uncertainties that arise during flight (e.g., bad weather, departure delays), automated systems will be used to detect conflicts, and provide resolution advisories to the pilot.

Although automation provides the resolutions, pilots will ultimately be responsible for the validity of that resolution. They should therefore be able to monitor the traffic situation, and the proper functioning of the automation, and should be able to intervene in case the automation fails. In other words, pilots should be able to detect, and act upon very infrequent situations that were not anticipated in the design of the automation. It is therefore of paramount importance for automation to be transparent and understandable to the operator [3]-[6].

The work presented in this paper is part of an ongoing study on the design of a 3-D separation assistance interface. The study employs a constraint-based approach, inspired by Ecological Interface Design (EID). EID is a proven design paradigm from the domain of process control [7], [8], that has in recent years also been applied in several aviation-related interface concepts [9]-[16]. In this approach, work-domain analysis tools such as the Abstraction Hierarchy (AH) are used to identify relevant constraints and relations on multiple levels of abstraction [17], [18]. An extensive analysis of the work

This work has been co-financed by the European Organisation for the Safety or Air Navigation (EUROCONTROL), under its Research Grant Scheme launched in 2008, and by the National Aerospace Laboratory NLR. The content of the work does not necessarily reflect the official position of EUROCONTROL or the NLR on the matter.

The authors are with the Control and Simulation section of the Faculty of Aerospace Engineering, Delft University of Technology, Kluyverweg 1, 2629 HS Delft, The Netherlands. Email: J.Ellerbroek@TUDelft.nl domain relevant to airborne separation was performed during the design of previous constraint-based concepts [12], [13], [16]. This paper will summarize the relevant constraints and relations that were identified in the analysis, which stands at the basis of the concept presented in this paper. For a more exhaustive description of the actual work-domain analysis, the reader is referred to the previous publications.

The aim of this study is to create an interface that realizes proper support for airborne separation, by showing the implications of other traffic for the affordances* of locomotion, and how they relate to limitations of the own aircraft [12], [13]. The interface presented in this study does not explicitly relate to specific automation functions. Instead, it visualizes work domain information, which invariably forms the premise on which both automation and the human operator should base their actions. By going beyond visualizations that relate only to the automation logic, these displays help pilots gain a deeper understanding of the functions and relations within the work domain [17], [18], [21], which will be invaluable to pilots when they need to judge the automation's functioning [22]. These displays should provide support in routine as well as unforeseen situations, where pilots may have to rely on their own problem-solving skills to resolve a conflict.

This study has led to three display concepts [12], [13], [16]. Each of these concepts presents a planar projection of the own aircraft's 3-D maneuver space. All three projections represent simplified, 2-D versions of the maneuver space. They inescapably discard information, a problem that is inherent to the presentation of multi-dimensional data on a 2-D surface [23], [24]. Our ultimate goal is therefore to find a representation that mitigates as much as possible the problems of presenting multi-dimensional data on a 2-D surface.

Several studies have investigated the effects of 3-D visualization methods for airborne traffic information displays [25]-[27]. They compare between perspective and (co-)planar displays that give a basic representation of traffic. For the current work, however, the focus lies not only on representing traffic, but also on what such traffic means to pilots in terms of achieving functional goals, and how it relates to other functions and constraints in the work domain. This will pose different demands on the method of presentation.

This paper argues for a co-planar representation, on the basis of previous concepts and the corresponding work-domain analysis. It will describe how to mitigate the problems that arise when information which is intrinsically three dimensional

\footnotetext{
* James J. Gibson defined affordances as opportunities for action, provided by an object or by the environment. An affordance is considered always in relation to the actor, and therefore dependent on the actor's capabilities and goals [19], [20]. For instance, with respect to an engine, air affords propulsion, but with respect to a wing, air affords lift.
} 
is distributed across two 2-D displays, and how to show the interactions that can occur between the two planar presentations. The decision for a co-planar display is a departure from the aim of the previous concept [16], which was to create a single, integrated perspective presentation of the 3-D constraints. The following section will therefore discuss the rationale for choosing a co-planar display. Section III introduces functional presentations for relevant work-domain constraints. The fourth section describes the co-planar display concept. A fifth section illustrates how the visualizations in the display concept link back to the work-domain analysis. The paper concludes with a discussion of the benefits, drawbacks, and remaining challenges of this concept.

\section{THREE-DIMENSIONAL DATA VISUALIZATION}

For the visualization of a 3-D space on a 2-D screen, two options can be distinguished: perspective displays and co-planar displays, each with their own benefits and drawbacks [23], [24]. The decision to choose either of these two methods will depend on the task requirements for the resulting display. A co-planar display has uniform, undistorted axes in its viewing planes, which benefits precise position and angle judgments. Perspective displays, on the other hand, have more "pictorial realism": they correspond more closely to the 3-D world [26], [28], [29]. Perspective displays can also employ texture and shading to increase realism, and improve spatial awareness [30]. Perspective displays might therefore be preferred when the task requires complex shape understanding. St. John et al. also differentiate between tasks that involve only separated spatial dimensions, and tasks that involve integrated spatial dimensions, where co-planar displays are better suited for the former, and perspective displays for the latter [24].

A drawback of co-planar displays in the current context is that some of the information on the interaction between locomotion constraints is lost, when these constraints are presented using separate horizontal and vertical projections. Also, distributing the information across two displays requires the pilot to mentally integrate the information from both displays. Perspective displays, on the other hand, suffer from perspective distortions, which can induce errors in judging distances and angles on the display [27], [31], [32]. The presentation of 3-D structures also suffers from problems of occlusion: when viewed from a fixed angle, the front facing side of the structure hides the internal details of the structure.

\section{A. Motivation for a co-planar display concept}

In the design of a separation assistance display concept, the choice between visualizations should depend on the specifics of the separation task, and how it is performed. From previous studies and experiments, several arguments can be found for the use of a co-planar display. First, experiments performed in this study, as well as other studies, showed that pilots have a strong preference for single-axis resolution maneuvers [26], [33]-[36]. Second, the design of previous constraint-based separation assistance displays illustrated that traffic constraints can become complex, yet precise judgment of these constraints is valuable for safe and efficient conflict resolution. They also illustrate that the planar projections of the constraints show an intuitive relation with the absolute geometry of the conflict, which benefits situation awareness. Perspective distortion makes this relation less visible in a perspective projection, a problem that also hampered the previous concept for a perspective 3-D interface [16]. Although that concept employed constant-velocity cutting planes to reduce the complexity of the constraint visualization, it did not reproduce the intuitive visual relation with the spatial representation of the conflict.

Two of the three current constraint-based separation assistance displays will be used as a basis for the co-planar concept [12], [13], [16]. The three current display concepts provide three orthogonal projections of the maneuver space: a topdown projection [12], a side-view projection [13], and a frontfacing, ego-centric equidistant cylindrical projection [16]. The first two are presented on the Horizontal Situation Display (HSD), and Vertical Situation Display (VSD), respectively. The third concept does not have an equivalent existing display in the cockpit. Because the first two concepts feature the most intuitive maneuver space projections, and as they correspond closest to current re-planning tasks and displays, these will be used in the co-planar display concept. Each of the two original display concepts assumes that a traffic conflict lies exactly in its plane, and the challenge in the design of the co-planar concept discussed in this paper lies in showing the interactions that exist between the projection planes of the coplanar display. This will be discussed in Section III.

\section{B. Comparison with other 3-D displays}

Although most current research on airborne separation assistance systems focuses on the development of automated systems that assist pilots with the separation task [37]-[41], there are also several display concepts have been developed as aids in the task of self-separation [39], [42]-[44]. There are two distinct types of conflict representation that are used in these displays: a spatial representation, which is similar to traditional situation displays, and a maneuver-space representation, i.e., visualizing how proximate traffic limits ownship maneuverability in terms of airspeed, heading, and vertical speed. Some displays use only one of these representations, others combine them. An important benefit of spatial representations is that they offer an intuitive overview of the situation, familiar to anyone who has ever used a map. Maneuver-space displays, on the other hand, are useful because they reveal a direct link between constraints or commands, and the applicable maneuver dimensions.

A second distinctive factor between displays is whether they show explicit (automated) commands, or constraints on maneuvering. Benefits of explicit command displays are that they suffer relatively little from display clutter, and that they reduce workload. Automation also has the potential of providing the most efficient conflict resolution options. The most important drawback of displays that show only explicit resolution commands is that they hide the rationale behind the automation. These displays do not support human information seeking, and, in case of automation failure, the pilot is not 
supported in recognizing failure, nor in seeking alternatives. In these situations, performance can even be worse than when completely unaided [22].

Constraint displays, on the other hand, give a continuous view on maneuver options and limitations, which allow pilots to evaluate their own resolution maneuvers. Depending on how constraints are visualized, these displays can show the structure of, and relations within the work domain, and can therefore provide a useful basis for illustrating automation logic. By showing higher level information and relations, these displays also allow pilots to investigate the validity of the data. An important drawback of constraint displays is that they can result in more display clutter, compared to showing only explicit commands. According to Tufte's views on the use of details ("To clarify, add detail"), however, this is not necessarily a drawback for a well designed display [45].

The existing concepts described in [39], [42], [44] show that the concepts presented in the current study are not the only displays that show constraints on maneuvering instead of only presenting explicit commands. The concept presented in [42] provides a spatial representation of constraints in the form of no-go areas on horizontal and vertical map displays. A different approach was taken in [39], where constraints are indicated with colored conflict-bands on the compass, the speed tape and the vertical speed indicator on the Primary Flight Display, the HSD, and the VSD. The concept described in [44] introduces a new display that presents constraints in a perspective, 3-D maneuver space.

The concepts in the current study aim to improve upon such constraint-based concepts by visualizing the structure of the work domain, and by illustrating the relations between lowerlevel elements and higher-level information. The remainder of this paper will describe how properties of the own aircraft and the surrounding traffic are related to each other, and to higherlevel constraints and functions, in a way that is made visually apparent on the display.

\section{FUNCTIONAL PRESENTATION OF CONSTRAINTS}

For airborne trajectory planning and self-separation, several relevant constraints have been identified [12], [13], [16]. These constraints fall broadly into two categories: constraints internal to the own aircraft, and constraints external to the aircraft.

The internal constraints relevant to the problem of separation relate to the various limitations on aircraft performance. In addition to these internal limitations, the maneuver space is further constrained by external factors such as weather, terrain, other traffic, and airspace boundaries (such as those from restricted airspace areas). For airborne separation, the focus obviously lies on the constraints imposed by other traffic.

Functional presentations of these constraints, and the relations between these constraints, should provide a description that is compatible to the means that are available to the controller. For trajectory planning, this implies that the description should relate the goals and affordances of the system, to inputs that match common flight practice. In cruise flight, pilots control their aircraft by manipulating velocity, track angle*, and altitude settings, using the autopilot or by modifying the planned route in the Flight-Management System (FMS). A successful separation assistance interface should relate these control variables and their operational limits to the affordances of the airspace.

\section{A. Velocity action space}

A modern glass cockpit supports trajectory planning through the Horizontal Situation Display and the Vertical Situation Display, which show horizontal and vertical projections of task-relevant information such as the planned route, terrain, weather, and other traffic. While these visualizations clearly identify the elements of the airspace that constrain the maneuver options of the aircraft, they do not show how these elements shape the possibilities for pilot action. Because the operator action space is not shown in a meaningful way, it remains difficult to relate higher level goals and constraints to the available actions and inputs.

The design philosophy employed in this study proposes to achieve this by combining the existing spatial representation of airspace elements, with a velocity action space, that relates own aircraft maneuver variables velocity, track angle and vertical speed, to the identified internal and external constraints [12], [16]. This action space is defined as the reachable subset of the 3-D vector space that contains all possible velocity vectors (i.e., all combinations of velocity, track angle and vertical speed).

When zero wind is assumed, an aircraft velocity vector in this vector space can be defined as follows:

$$
\mathbf{V}=V_{T A S} \cdot\left[\begin{array}{c}
\cos (\chi) \cos (\gamma) \\
\sin (\chi) \cos (\gamma) \\
\sin (\gamma)
\end{array}\right]
$$

where $V_{T A S}$ is the (true) airspeed of the aircraft, $\chi$ the track angle, and $\gamma$ the flight-path angle, or climb angle of the aircraft. Vertical maneuvering is more commonly expressed in vertical speed, which can be derived from $V_{T A S}$ and $\gamma$ : $V S=V_{T A S} \sin (\gamma)$. The presence of wind can be of influence on the velocity action space representation, but only when ownship and intruder aircraft experience significantly different wind conditions. It is therefore kept out of the current analysis.

\section{B. Internal constraints}

The reachable area that defines the velocity action space is bounded by constraints that are internal to the own aircraft. These constraints relate to the various limitations on the performance of the aircraft, such as bank limits, maneuver dynamics, available engine thrust, stall, structural considerations, buffet characteristics, and requirements on productivity, emissions and passenger comfort. These limitations result in several constraints relevant to the task of trajectory planning, such

\footnotetext{
${ }^{*}$ Track angle differs from aircraft heading in the presence of wind. Heading indicates in which direction the aircraft nose is pointing, as indicated e.g. on the magnetic compass. The track angle gives the direction in which the aircraft is flying. With no wind, these angles are equal. With cross-wind, however, there will be an offset between heading and track (the drift angle).
} 
as maximum turn rates, maximum and minimum operating speeds, fastest and steepest steady climb and descent, and the steepest steady climbing and descending turn. Some of these constraints also show interactions: For example, in turning flight, increasing the bank angle will also affect the minimum velocity and the maximum attainable climb angle [46].
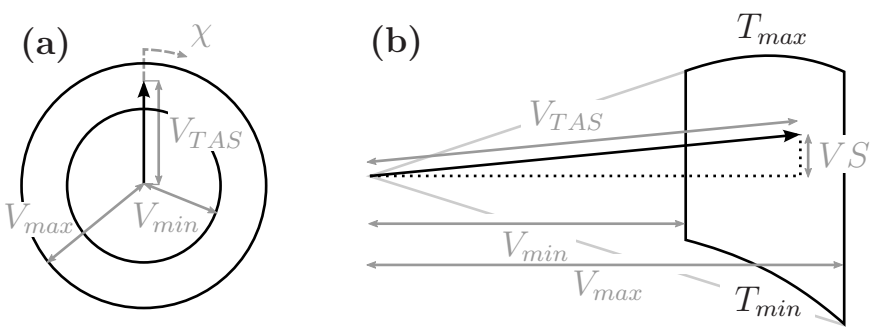

Fig. 1. The 3-D velocity action space illustrated in two planar projections. (a): Horizontal maneuvering can be expressed in combinations of airspeed $\left(V_{T A S}\right)$ and track angle $(\chi)$. The minimum $\left(V_{\min }\right)$ and maximum $\left(V_{\max }\right)$ obtainable airspeeds are the main constraints for horizontal maneuvering. (b): Vertical maneuvering can be expressed in combinations of airspeed ( $\left.V_{T A S}\right)$ and vertical speed $(V S)$. It is also constrained by the minimum $\left(V_{\min }\right)$ and maximum $\left(V_{\max }\right)$ obtainable airspeeds. Aircraft performance with maximum $\left(T_{\max }\right)$ and minimum $\left(T_{\min }\right)$ thrust settings determine maximum steady climb and descent, respectively.

Fig. 1(a) and Fig. 1(b) illustrate how these constraints can be visualized in the horizontal and vertical plane, respectively. The horizontal maneuver space is shaped by the limitations on airspeed, which reduce it to the ring-shaped area in Fig. 1(a).

Vertical maneuvering is also constrained by airspeed limitations, as well as by the steady climb and descent performance. Fig. 1(b) shows how these constraints combine in the vertical plane. The vertical edges of the action space result from the limits on airspeed. The curved edges at the top and bottom of the vertical action space visualize the maximum obtainable steady climb and descent at each velocity, respectively. The resulting contour is also known as the flight envelope of the aircraft (refer to [13] for the derivation of this contour).

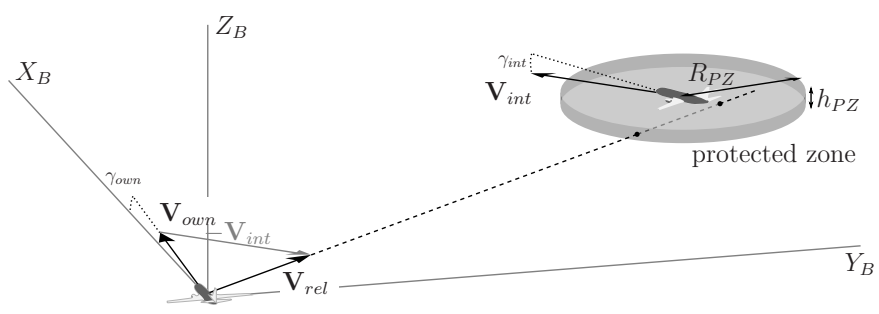

Fig. 2. A conflict situation. Ownship and intruder are in conflict when the line that extends from the relative velocity vector $\left(\mathbf{V}_{r e l}\right)$ crosses the intruder Protected Zone (PZ). The PZ is a flat, 3-D disc around each aircraft, that should remain clear of other traffic. Common dimensions for this $\mathrm{PZ}$ are $R_{P Z}=5 \mathrm{nmi}$ and $h_{P Z}=2,000 \mathrm{ft}$.

\section{External constraints}

In unmanaged airspace, the reachable area that is enclosed by the internal aircraft constraints is further restricted by several external factors, such as weather, terrain, and traffic. For a self-separation interface the focus obviously lies on the constraints imposed by other traffic. These traffic constraints are shaped by a minimum horizontal and vertical separation between any two aircraft, that should be adhered to at all times. With common values of 5 nautical miles horizontal, and 1,000 feet vertical separation, this results in a 3-D Protected Zone $(P Z)$ : A flat, 3-D disc around each aircraft, that should remain clear of other traffic (illustrated for the intruder aircraft in Fig. 2) [33], [47]. Intrusion of this space is referred to as a loss of separation. A conflict is defined as a predicted future loss of separation, within a certain time horizon [48]. For this concept, the time horizon was set between 60 seconds and 10 to 20 minutes [16].

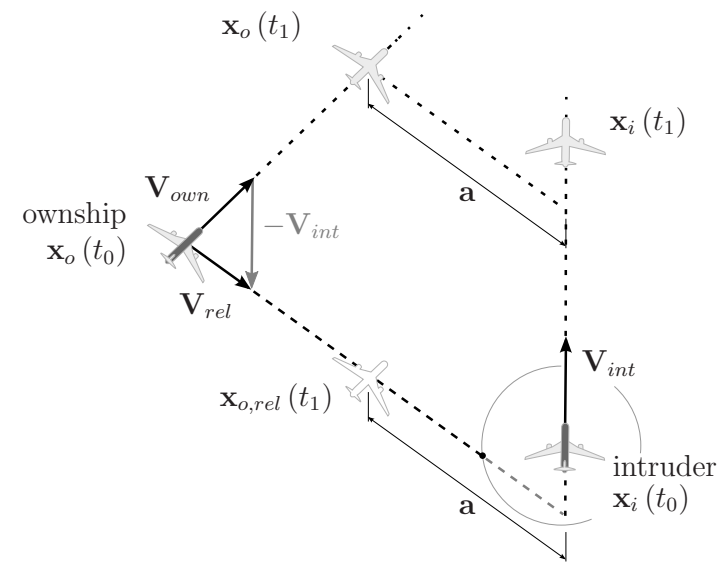

Fig. 3. Relation between ownship motion relative to the intruder, and the absolute motion of ownship and intruder. Ownship and intruder are shown at time $t_{0}$ and $t_{1}$. $\mathbf{x}_{o, r e l}$ shows how ownship has moved relative to the intruder. Line a shows that the change in orientation between ownship and intruder along the relative path is equal to the change along the absolute paths.

Although ownship and intruder intent can influence the constraints imposed by the intruder, the current study will only employ the current states to derive these constraints. Previous studies did incorporate the effect of intent on maneuver affordances [49]-[51], however, this is beyond the scope of the current study. Under the assumption that intruder and ownship state remain unchanged in the near future, a conflict can be predicted using the speed of ownship, relative to the intruder:

$$
\mathbf{V}_{\text {rel,own }}=\mathbf{V}_{\text {own }}-\mathbf{V}_{\text {int }}
$$

Relative velocity vector $\mathbf{V}_{\text {rel,own }}$ indicates how ownship moves with respect to the intruder aircraft ${ }^{*}$, see Fig. 3 . When the relative track of ownship (the line extended from the relative velocity vector) crosses the intruder protected zone, a loss of separation will occur in the near future, see Fig. 2.

For any given traffic geometry, a set of relative velocity vectors $\mathcal{V}_{F A}$ can be defined that would result in a conflict between the involved aircraft (i.e., all possible relative velocity vectors where the resulting relative tracks cross the intruder PZ). Fig. 4 gives an illustration of $\mathcal{V}_{F A}$. It is a construct of two slanted cones, connected by straight sections. Both cones have their apex at the ownship location, and their curvature is

\footnotetext{
${ }^{*}$ Note that the relative motion of intruder symbols on a traffic display is opposite to this relative velocity: There, the own aircraft symbol is standing still, and intruder aircraft symbols move relative to the own symbol on the display. The current concept observes relative motion from the perspective of ownship $\left(\mathbf{V}_{\text {rel,own }}\right)$ in order to find constraints on own maneuvering.
} 


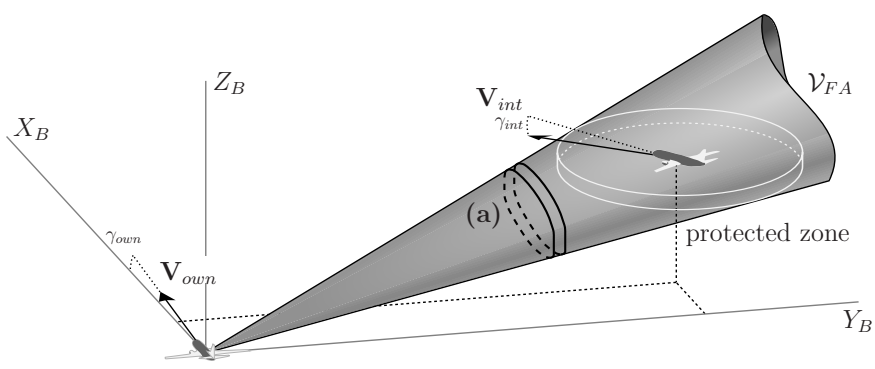

Fig. 4. The 3-D forbidden area $\mathcal{V}_{F A}$ consists of a conical area that is aligned with the edges of the intruder protected zone, as seen from the ownship, and has its apex situated at the center of ownship. Cross-section (a) shows how the thickness of the protected zone changes the shape of the forbidden area from what would otherwise be a pure slanted cone, to a combination of two slanted cones, connected by a straight section.

aligned with the upper and lower circles of the intruder PZ. Cross-section (a) in Fig. 4 illustrates the effect of the thickness of the intruder protected zone: if the PZ had been flat, $\mathcal{V}_{F A}$ would have been a single slanted cone, and cross-section (a) would have been an ellipse. The thickness of the PZ introduces straight sections in the shape of $\mathcal{V}_{F A}$, and in cross-section (a).

The 3-D vector set $\mathcal{V}_{F A}$ marks the constraints that other traffic imposes on ownship (relative) motion with respect to that intruder aircraft, and will be referred to as the 3-D forbidden area in the remainder of this paper. This representation only varies as a function of time ${ }^{\dagger}$. This means that for the current time, the 3-D forbidden area represents the complete set of relative velocities that would result in a loss of separation. (a)

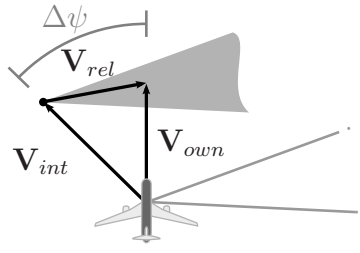

\section{(b)}

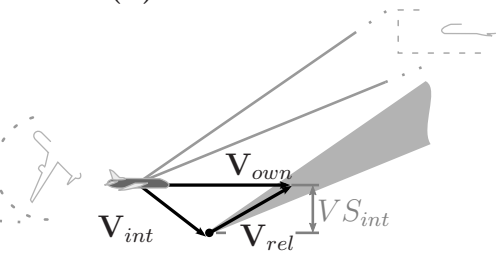

Fig. 5. Constraints on ownship velocity, expressed in an absolute velocity field. (a) illustrates horizontal intruder constraints, (b) illustrates vertical constraints. ownship and intruder are in conflict when the tip of the ownship velocity vector is inside the 3-D forbidden area. The apex of the shifted area corresponds with the intruder velocity vector, and can be used on the display to determine for instance relative track, and intruder vertical speed. The forbidden area bisector shows relative bearing of the intruder.

The concepts preceding the current study illustrated that the relation between the forbidden areas and the own velocity vector can be made visible by translating the forbidden area and relative velocity vector by the intruder velocity vector, see Fig. 5 [12], [16]. This would be equivalent to adding $\mathbf{V}_{\text {int }}$ on both sides of the equal sign in Eq. (2). The shifted forbidden area represents the constraints imposed by an intruder aircraft in a way that directly relates to the ownship maneuver options. An added benefit, illustrated in Fig. 5, is that the apex of the shifted forbidden area corresponds with the intruder velocity

\footnotetext{
${ }^{\dagger}$ When neither aircraft maneuver, the opening angle of the forbidden area will expand or contract only as a function of the closing speed of the intruder aircraft, with respect to ownship. When ownship and intruder are not on a collision course, the orientation of the forbidden area will also change as both aircraft pass each other.
}

vector, and that the direction of the bisector of the forbidden area is equal to the relative bearing of the corresponding intruder, properties that can be useful when assessing a conflict.

Note that this derivation of the forbidden area assumes instant state changes ${ }^{\ddagger}$. It can be shown that this is a safe assumption when a predicted conflict is still in the far future [52], [53]. However, maneuver dynamics will start to play a larger role when conflicts become more imminent: in the case of tactical maneuvers (within 10 minutes of a predicted conflict), unmodeled dynamics will cause significant errors, particularly speed maneuvers [52]. The previous horizontal display concept compensated for this by observing the constraint area at time $t_{c u r}+t_{\text {turn }}$. Here, $t_{\text {turn }}$ is the maneuver duration for the heading solution that corresponds to the respective forbidden area leg. A new relative position is extrapolated using the current aircraft velocities, which in turn is used to calculate the corrected position for the forbidden area leg. This method can also be applied to the current concept.

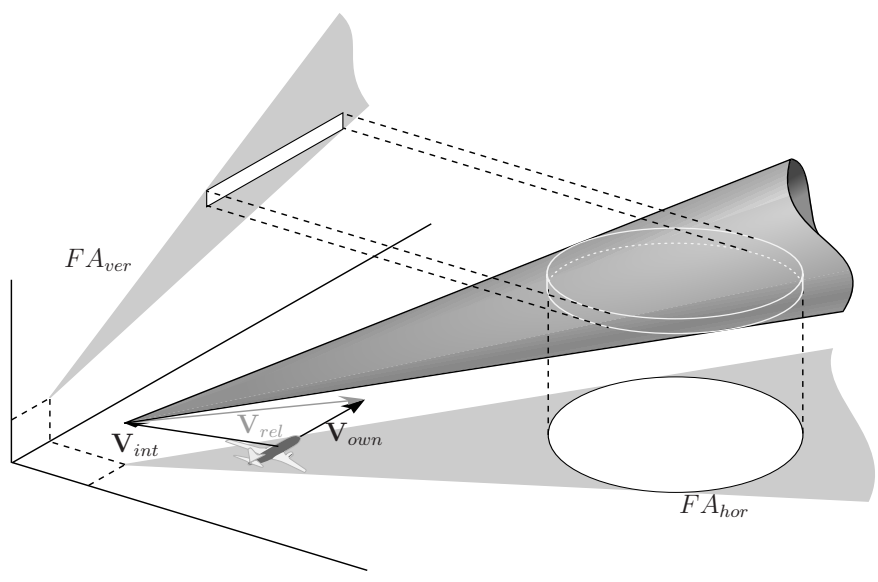

Fig. 6. Translated 3-D forbidden area and horizontal and vertical projections. $F A_{h o r}$ is the horizontal projection of the 3-D forbidden area, and presents constraints for ownship track and horizontal velocity. $F A_{v e r}$ is the vertical projection of the same area, and presents constraints for vertical motion.

\section{Planar constraint projections}

There are several visualization techniques for 3-D constructs such as the forbidden area, each with its specific benefits and drawbacks [23]. The drawback these methods have in common, however, is loss of context. This, however, is unavoidable, as some form of reduction is necessary to present multi-dimensional data on a 2-D surface [23], [24]. The challenge is to determine crucial parts of the context, and to find a visualization that maintains the relevant information.

The previous horizontal and vertical constraint displays both show an orthogonal projection of the 3-D forbidden area (illustrated in Fig. 6). These projections take the 3-D shape, and discard the coordinate that is orthogonal to the projection plane. The resulting shapes are triangular, with the triangle

$\ddagger$ In reality, a heading or speed change that is taken to resolve a conflict will take a certain amount of time. In that time, the constraint area will have grown slightly, and it might occur that what initially seemed a valid solution, will in fact not resolve the conflict, due to the expansion of the constraint area during the course of the maneuver. 
apex at the (projected) ownship position, and the triangle legs aligned with the edges of the projected protected zone.

In Fig. 6, $F A_{\text {hor }}$ is the horizontal projection of the 3-D forbidden area, and presents the constraints on relative track and horizontal velocity, for all values of vertical speed combined. $F A_{v e r}$ is the vertical projection of the 3-D forbidden area, and presents the constraints on relative vertical motion, for all values of the ownship track angle combined.

The orthogonal projections, then, provide a global contour of the forbidden area in their respective dimensions. Visualization of these contours has many benefits. Most importantly, the relation between the triangular contour and the geometry of the conflict is easily interpretable. The triangles reveal several key parameters of a conflict, such as spatial proximity, intruder bearing, intruder heading and velocity, and relative velocity, and how these parameters relate to each other. These cues also help to relate the forbidden areas to the traditional intruder symbols on the display [36].

However, because the projections show constraints for all values of the orthogonal coordinate, the constraints can be conservative when the conflict does not lie exactly in that plane. For instance, a certain combination of speed and track angle may lie in a horizontally projected forbidden area, but can still be conflict-free if there is enough vertical separation, or if vertical separation is obtained before a horizontal loss of separation occurs. This distinction cannot be made with the projected forbidden areas alone.

\section{E. Interactions between projection planes}

What these projections fail to show, then, is how the orthogonal planes interact with each other. The horizontal projection does not reflect vertical separation and maneuvering, and vice versa. Cutting plane visualization partly reveals this interaction, by showing a part (a 'slice') of the 3-D shape, for a given constant value of the third dimension [23]. In combination with the planar projection of the 3-D shape, it reveals which part of the projection is valid, for a specific point along the dimension that is orthogonal to the projection plane. The result of a cutting-plane intersection will therefore always be a subset of the planar projection of the 3-D shape.

Fig. 7 shows a horizontal cutting plane, that intersects with the 3-D constraint area for a certain given value of ownship vertical speed, i.e., the vertical offset of the cutting plane is equal to ownship vertical speed. The resulting 'slice' of the constraint area represents horizontal velocity constraints, taking into account the relative vertical motion and orientation. This reduced forbidden area is a subset of the horizontal projection of the 3-D constraint zone (also illustrated in Fig. 7). When ownship and intruder are not vertically separated, and the relative vertical speed is equal to zero, the horizontal reduced forbidden area will be equal in shape and size to the projected horizontal forbidden area.

Fig. 8 shows a vertical cutting plane, aligned with the ownship track, that intersects with the 3-D constraint area. The resulting slice of the constraint area presents vertical velocity constraints, taking into account how a conflict is oriented in the direction orthogonal to the ownship track (i.e., intruder crosstrack distance and relative intruder track angle). This reduced

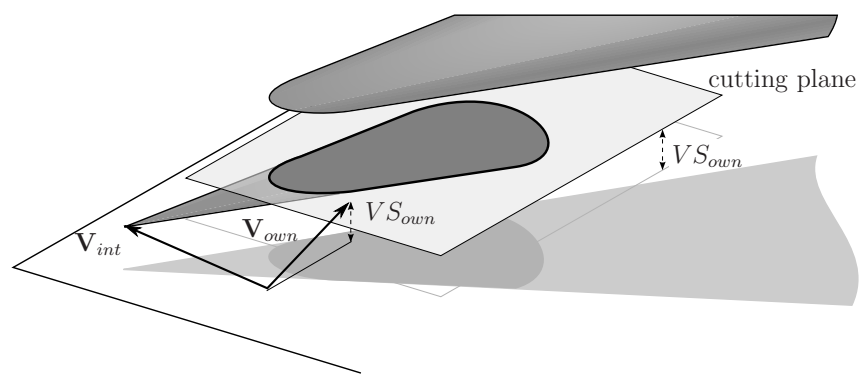

Fig. 7. The horizontal reduced constraint area is given by the intersection of the 3-D constraint area, and a horizontal cutting plane, offset vertically by the ownship vertical speed. The horizontal reduced area illustrates exact constraints on horizontal maneuvering, also for conflicts with non-zero relative vertical distances and velocities.

forbidden area will always be a subset of the vertical (sideview) projection of the 3-D constraint zone (see also Fig. 8). When ownship and intruder are flying with zero cross-track distance and relative track angle $\Delta \chi=180^{\circ}$ or $\Delta \chi=0^{\circ}$ (ownship and intruder are flying head-on or are overtaking, respectively), the vertical reduced forbidden area will be equal in shape and size to the projected vertical forbidden area.

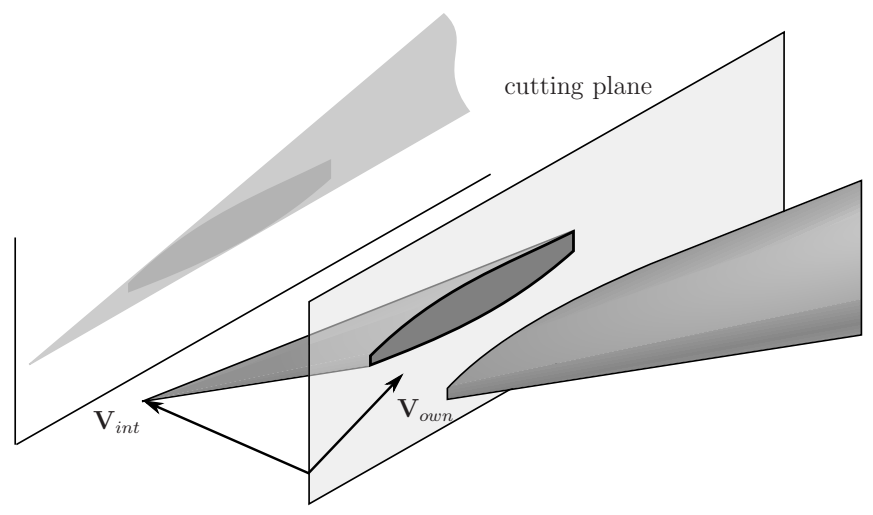

Fig. 8. The vertical reduced constraint area is given by the intersection of the 3-D constraint area, and a vertical cutting plane, aligned with the ownship track. The vertical reduced area illustrates exact constraints on vertical maneuvering, also for conflicts with non-zero cross-track distances and velocities.

Because these reduced forbidden areas depend on the variable that is perpendicular to their plane of projection, they effectively reveal an important part of the interaction between the planes of projection. The reduced areas are invariant with respect to state changes that lie in their own plane of projection, and therefore provide a consistent set of 2-D constraints, under the assumption that the third (perpendicular) variable is kept constant. In this case, the reduced areas only vary with time, similar to the forbidden area projections.

When the perpendicular variable is varied, the reduced area will always change in a predictable fashion. If the change in that variable is away from the tip of the 3-D forbidden area, the reduced area will also move away from the tip of the triangle in its respective projection. Similarly, if the change moves the velocity vector closer to the tip of the 3 -D forbidden area, the reduced area will move towards the tip of the triangle in its respective projection. Also, because the planar projections 

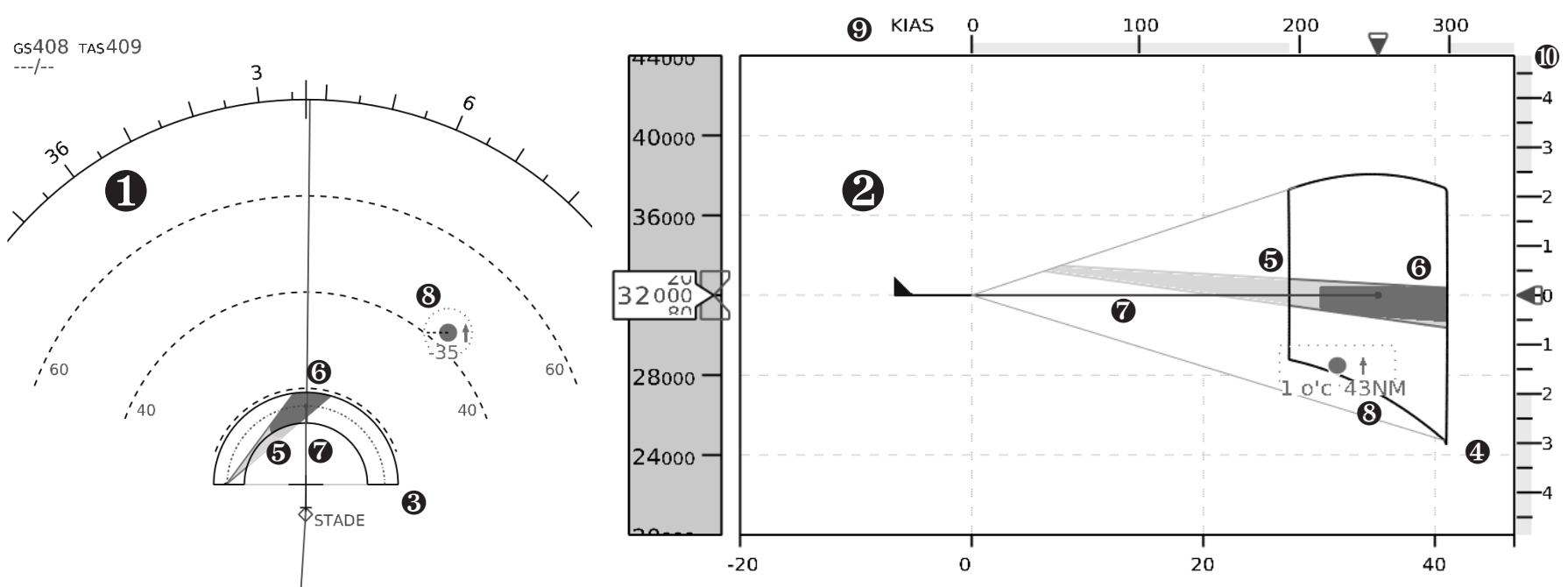

Fig. 9. Concept for a co-planar separation assistance display. This figure shows a Horizontal Situation Display (1) and a Vertical Situation Display (2), with added separation assistance overlays. $\mathbf{3}$ and $\mathbf{4}$ are the horizontal and vertical State-Vector Envelope, respectively. $\mathbf{5}$ is the projected forbidden area both on the HSD and the VSD. 6 is the reduced forbidden area on both displays. 8 Represents the ownship state vector, 8 is a TCAS-symbol indicating the relative location of the intruder aircraft. $\boldsymbol{\Theta}$ is a speed tape, showing current IAS, selected IAS, and simplified traffic constraints. (ID) is a vertical speed tape, showing the current and selected vertical speed in feet per minute, and simplified traffic constraints.

effectively result in a 2-D contour of the forbidden area at its widest point, projections of the reduced areas can never extend beyond the boundaries of the projected forbidden area.

Aside from interactions that result from shortcomings of methods for showing 3-D data on multiple, 2-D surfaces, interactions can also be found between several aircraft locomotion variables, and their limits. A very direct interaction can be found between the aircraft's bank angle, and its climb performance and stall speed: Increasing the bank angle will increase the stall speed, and reduce the maximum climb angle. On a larger time scale, changes in altitude will have an effect on minimum and maximum operating speeds, and on climb and descent performance [46].

These interactions can partly be captured in the visualization when the visualized constraints are dynamically calculated for the current values of the flight variables that influence it. There are, however, situations possible where dynamically calculated constraints do not suffice. Consider for instance a situation where a traffic conflict is solved by assuming a vertical speed that is close to the maximum climb performance. It can happen that although initially this climbing solution seemed to be a valid solution, the reduction in climb performance due to increasing altitude causes this solution option to disappear. This is, however, beyond the scope of the current study.

\section{CONCEPT}

Fig. 9 illustrates a design concept for a separation assistance interface, that presents separation-related affordance cues on a co-planar display. The combination of a Horizontal Situation Display and a Vertical Situation Display was chosen for this co-planar display concept, because these displays are omnipresent in the modern flight deck. These two displays also provide the most intuitive maneuver space projections, and they correspond closest to current re-planning tasks.

In this display concept, the 3-D traffic situation is visualized in two orthogonal, 2-D views: a top-down view (1), and a side view (2). Both views present an ownship-centered moving map, that shows spatial information such as the FMS route and intruder aircraft positions. In addition, constraints on ownship maneuvering are shown on both displays through velocity action-space overlays $(\mathbf{3}, \mathbf{4})$.

The top-down view presents information in a polar coordinate system: spatial information is expressed in relative bearing and distance, and the velocity action-space shows constraints for combinations of track angle and airspeed. The side view uses a Cartesian coordinate system: spatial information is expressed in along-track distance and relative altitude. Here, the velocity action-space shows constraints for combinations of airspeed and vertical speed.

\section{A. Traditional display elements}

The moving-map presentations on the HSD and VSD are not new: the HSD is present in all modern cockpits, and also the VSD is becoming more common. To match current practice, intruder aircraft are represented on both displays using TCASlike symbology* (8) [54]: an unfilled diamond indicates a nonconflicting intruder, a filled diamond indicates a conflicting intruder, with more than five minutes to a loss of separation. This is considered a low-priority conflict. A conflict is considered medium priority when a loss of separation is between three to five minutes away, indicated with a solid circle as intruder symbol. A high priority conflict is less than three minutes away, and is indicated with a solid square.

Separation margins are indicated around each intruder on both displays, which results in a circle on the HSD, and a flat rectangle on the VSD, see Fig. 10. On the HSD, the intruder's horizontal speed vector is shown with a dotted trend line. The length of this line is scaled such, that it equals the radius of

\footnotetext{
${ }^{*}$ Note that this is not necessarily the best intruder visualization. Intruder symbology design, however, is beyond the scope of the current study. TCAS symbology was therefore chosen to match current practice.
} 
(a)

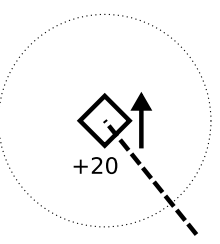

Fig. 10. Intruder aircraft symbology. Intruders are visualized using TCASstyle symbology: unfilled diamonds for non-conflicting aircraft, filled diamonds for low-priority conflicts, filled circles for medium-priority conflicts, and filled squares for high-priority conflicts. (a): Intruder symbology as shown on the HSD. The TCAS symbol is shown together with the separation margin, a speed vector, a vertical speed arrow, and a flight-level offset. (b): Intruder symbology as shown on the VSD. The TCAS symbol is shown together with the separation margin, a vertical speed arrow, the relative bearing in hours o' clock, and the intruder distance in nautical miles.

the separation circle if the horizontal speed of the intruder is equal to the ownship horizontal speed.

A small up/down arrow is shown next to the intruder symbol, when the vertical speed of that intruder exceeds 500 $\mathrm{ft} / \mathrm{min}$. A signed number below the intruder symbol indicates the vertical offset in flight levels (1 flight level equals 100 feet), see Fig. 10(a). On the VSD, the intruder TCAS symbol is accompanied by a label that shows the relative bearing in hours o' clock, and the distance in nautical miles. An up/down arrow is shown to the right of the TCAS symbol when the vertical speed of that intruder exceeds $500 \mathrm{ft} / \mathrm{min}$, see Fig. 10(b).

In addition to the map view, the vertical display also includes a speed tape, and a vertical speed tape. The speed tape $(\boldsymbol{\theta})$ shows current Indicated Air Speed (IAS), selected IAS, and simplified speed constraints in knots. The vertical speed tape (ID) shows the current and selected vertical speed, and simplified vertical speed constraints, in feet per minute.

\section{B. Velocity action-space overlays}

The horizontal State-Vector Envelope (SVE) (3) shows the affordance space for horizontal maneuvering in terms of track angle and airspeed, see Fig. 11(a). Because a horizontal situation display in expanded mode (as in Fig. 9) does not show traffic behind the own aircraft, the horizontal state-vector envelope also shows only solutions with $|\Delta \chi| \leq 90^{\circ}$. Current horizontal situation displays also have modes that show the situation behind the ownship. In such a mode, the horizontal state-vector envelope would be shown as a whole circle. The vertical State-Vector Envelope (4) is illustrated in Fig. 11(b), and shows the affordance space for vertical maneuvering in terms of airspeed and vertical speed.

Intruder aircraft that are within detection range will further reduce the available maneuver space in the horizontal and vertical State Vector Envelopes. The reduced forbidden areas (6), derived in the previous section, give the most precise representation of these constraints, see Fig. 11. On the HSD, a reduced forbidden area gives the constraints imposed by an intruder on ownship track angle and airspeed, for the current value of ownship vertical speed. On the VSD, a reduced forbidden area gives intruder-imposed constraints on ownship airspeed and vertical speed, for the current ownship heading.

Note that each intruder adds a forbidden area to the available maneuver space. These forbidden areas, however, work in a cumulative fashion: selecting a 'clear' area solves all detected conflicts, without creating a new conflict. In the current concept, the derivation of the constraint areas uses only state information, and will therefore only be valid when there are no imminent trajectory changes. Although the influence of intent information has been considered in previous concepts [49][51], this is out of the scope of the current study.

The reduced forbidden areas result from the intersection between a flat cutting plane, and the 3-D forbidden area: a compound of two slanted conical shapes, aligned with the top and bottom of the intruder protected zone. The shapes that result from this intersection range from circles, to ovals, to open-ended hyperbolic curves, see Fig. 11.

Ref. [36] describes how the triangular shapes that result from planar projection of the forbidden area can be used to derive several relevant cues about the spatial configuration of a conflict. These cues make it easier to relate forbidden areas to intruder symbols on the map view, but also provide more information on the urgency of a conflict, and how different resolutions would affect the traffic situation. In other words, while the reduced forbidden areas provide more precise constraint visualization, they sacrifice important cues that link several display elements together. The current concept therefore combines the reduced forbidden areas with the outline of the corresponding projected forbidden areas (5). In addition to the added situational information, these outlines also provide an outer limit on the shape and size of the reduced forbidden area, when a perpendicular flight parameter is modified.

The current ownship airspeed, track angle and vertical speed are presented on the two displays by two velocity vectors $(\boldsymbol{O})$ that extend from the origin of the SVE to a certain point in the velocity vector space. On both displays, the length of these vectors is equal to the ownship airspeed. On the vertical situation display, the ownship vertical speed results in a rotation $\gamma=\arcsin \left(V S / V_{T A S}\right)$ of the velocity vector. Because the horizontal situation display is oriented track-up, the horizontal velocity vector has a fixed, vertical orientation. A dot, four kts wide, attached at the tip of each velocity vector, acts as a margin for maneuver selection [36].

In combination with the reduced forbidden areas, the velocity vectors show the affordance of avoidance: placing either of the velocity vectors outside all of the forbidden areas results in a conflict-free trajectory. Intruder flight-path vectors are also shown as dots at the tip of the corresponding forbidden area triangle. On the horizontal display, the distance from the tip of a triangle to the center of the SVE is equal to the airspeed of the corresponding intruder, see Fig. 11(a). On the vertical display, however, this distance is equal to the in-track component of the intruder airspeed, $V_{\text {vert }, \text { int }}=V_{T A S, \text { int }} \times \cos \left(\chi_{\text {int }}-\chi_{\text {own }}\right)$. Here, $V_{T A S, \text { int }}$ is the intruder airspeed, $\chi_{\text {int }}$ is the intruder track angle, and $\chi_{\text {own }}$ the ownship track angle. Moving the ownship velocity vector towards one of these dots to resolve a conflict will lead to a very inefficient resolution, as it will cause ownship to fly parallel to the intruder [12].

\section{Conflict urgency visualization}

In addition to varying intruder symbology, conflict urgency is also indicated using color coding for all of the display 

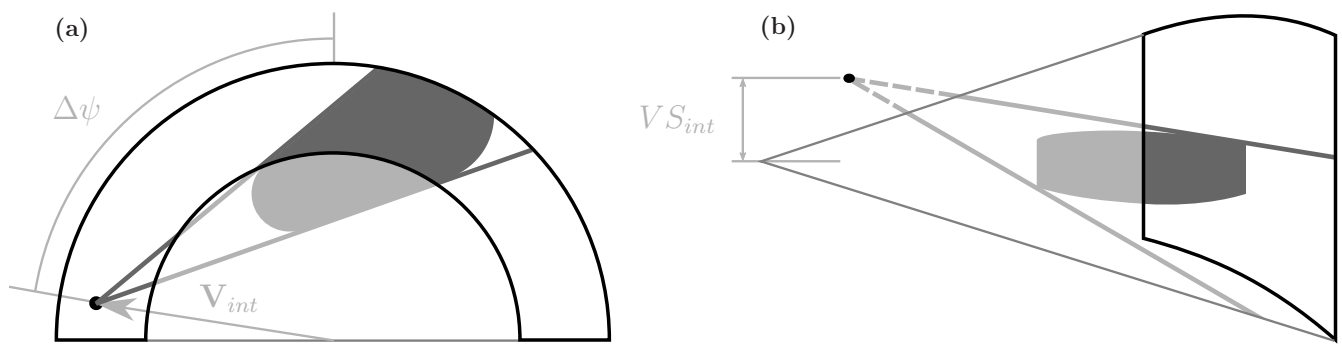

Fig. 11. The horizontal (a) and vertical (b) State-Vector Envelopes. The forbidden areas correspond to one intruder, who is situated below, to the front and to the right of ownship, crossing ownship from right to left, and climbing at a shallow climb angle. (a): The circular boundaries that constrain the horizontal maneuver space represent the aircraft minimum and maximum operating speeds. The intruder track is offset from the ownship track by $\Delta \psi$. The triangle apex represents the intruder velocity $\mathbf{V}_{\text {int }}$. (b): The vertical maneuver space is bounded by minimum and maximum operating speeds, and by minimum and maximum steady-state climb. The vertical offset of the triangle apex corresponds to the intruder vertical speed, $V S_{i n t}$.

elements that correspond to one intruder. This means that the aircraft symbols on both displays, as well as the forbidden area triangles and reduced forbidden areas on both displays are colored according to the urgency of the conflict between ownship and the corresponding intruder. Similar to the TCAS system, four levels of urgency have been defined for the current concept [54]. The lowest urgency level corresponds with intruder aircraft that are currently not in conflict with ownship. For these intruders, the display elements are colored white. The second level of urgency corresponds with a conflicting intruder, with a loss of separation further than five minutes away. This is defined as a low-urgency conflict, and display elements are colored yellow. A medium-urgency conflict corresponds with a loss of separation between three and five minutes, and is colored orange on the display. A highurgency conflict indicates a loss of separation within less than three minutes, and is colored red on the display.

\section{Visual momentum}

When more than one intruder aircraft needs to be shown on the display, it becomes more important for the display to provide ways to link the several visual elements on the display to an intruder and to each other. Visual cues that facilitate this link increase visual momentum: they facilitate the integration of information across multiple displays, and between elements on each display [55]. This integration is essential for proper SA, as the elements on both displays are intrinsically tied together. Manipulation in one plane will often affect constraints in the other plane. Visualizing which elements belong together should aid pilots when assessing these relations.

Woods introduces functional data overlap as a method that "improves the visual momentum across multiple displays by 'presenting pictorially the functional relationships that cut across display frame boundaries' ". In other words, visual momentum can be improved by showing particular information on both displays, and by visualizing relationships between information on both displays. The color coding that is used to indicate conflict urgency is an obvious way to improve visual momentum. The shape and orientation of the conflict zones, however, also provide ways to identify display elements that belong together. Examples are the distance to an intruder, which also determines the opening angle of the corresponding forbidden areas, the predicted intruder flight path determines the location of the tip of the horizontal triangle, and the vertical speed, that determines the vertical position of the tip of the triangle on the vertical display.

\section{E. Comparison with previous concepts}

The main difference between the current co-planar display concept and the previous separate horizontal and vertical display concepts, is the visualization of the interactions that can occur between the planes of projection. The horizontal display shows constraints on horizontal maneuvering, under the assumption that intruding aircraft are flying level, within minimum vertical separation. Similarly, the vertical display shows constraints on vertical maneuvering, assuming zero cross-track distance and maneuvering. These projected constraints become increasingly conservative when conflicts deviate from these assumptions. The reduced forbidden areas show more precise constraints by taking the conflict orientation orthogonal to each projection into account.

Fig. 12 illustrates how the constraints imposed by an intruding aircraft change when the corresponding conflict geometry can no longer be defined in a single plane of projection. All four examples in Fig. 12 show how conflict constraints would be visualized on the new display. Note that on the original two displays the visualization would be similar, but that all triangles would always be completely filled.

The first conflict, shown in Fig. 12(a), corresponds to an intruder that is both on the same track and the same level as ownship, and both aircraft are flying level. In this case, neither the assumptions for the original horizontal display, nor those for the original vertical display are violated. As a result, the constraints imposed by the intruder are presented as completely filled triangles on the new display, completely identical to what the visualization for this conflict would be on the original displays.

Fig. 12(b) shows how the constraints change when the intruding aircraft starts to climb. Because the intruder is still on the same track as ownship, the assumptions for the original vertical display still hold, and the presented vertical constraints are still identical to how they would be presented on the original vertical display. The horizontal constraints, however, change as a result of the vertical maneuver of the intruder. Where the original horizontal display would show a conflict, 


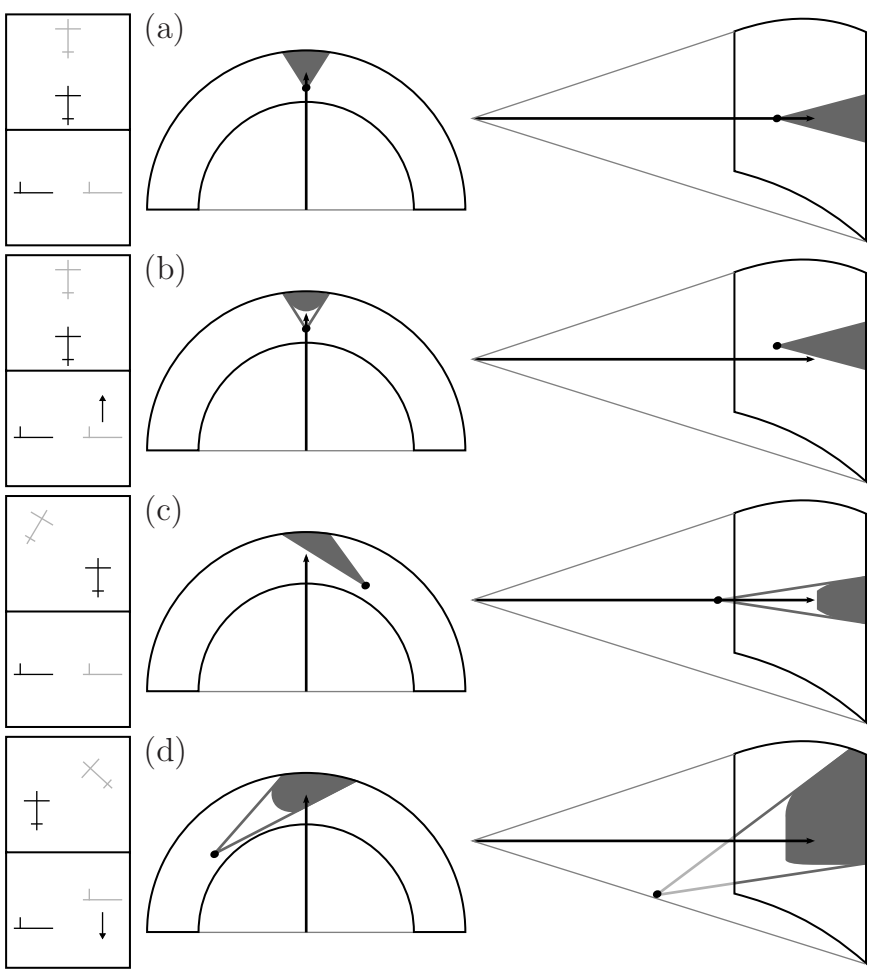

Fig. 12. Example scenarios. An overview of each scenario is given on the left. The black aircraft symbols represent ownship, the gray symbols represent the intruder. (a) Ownship is behind and overtaking the intruder, both are flying level, at equal altitude. (b) Intruder is climbing. (c) Both are flying level, but the intruder is to the left of ownship, crossing to the right. (d) Intruder is to the right of ownship, crossing to the left, descending from a higher altitude.

the reduced forbidden areas reveal that ownship would have to accelerate to get into conflict with the intruder.

Fig. 12(c) shows how the vertical constraints change when the intruder is on a different track than ownship. Similar to the situation in Fig. 12(b), the original vertical display would show a conflict, while in reality the intruder passes in front of ownship before they get too close. Fig. 12(d) shows that both the horizontal and the vertical presentation of constraints change when the intruder is both off level and off track. In this example ownship and intruder are still in conflict. The presentation in Fig. 12(d) shows that maneuvers exist that solve the conflict, while still being in both the horizontal and vertical triangles. Such solutions would be impossible to derive from the original two displays.

\section{RELATIONSHIPS BETWEEN THE AH AND THIS CONCEPT}

The constraint-based approach that was adopted in this study used work-domain analysis tools such as the Abstraction Hierarchy to identify constraints and relationships on multiple levels of abstraction [17], [18]. Although the work-domain analysis for this concept has not changed significantly since the previous concept, looking at how the visualizations in the current concept are related to that work-domain analysis can provide a useful review of the concept, as well as relevant insights for future design iterations and experiments. This section will therefore briefly describe how the different elements of the display link back to the functions, constraints and relationships in the abstraction hierarchy (Fig. 1 in [16]). Ref. [16] describes the work-domain analysis in more detail.

The velocity action-space overlays proposed in the display concept form the main additions to the horizontal and vertical situation displays. These overlays give a consistent view on the relations between locomotion inputs and the primary functions of productivity, efficiency and safety, and they show how these relationships are influenced by several identified constraints.

Together with the velocity vectors, the horizontal and vertical state-vector envelopes relate to the safety goal, by showing how internal constraints (available power, structural limits, ...) limit possible velocity vectors. In combination with the horizontal FMS track on the map display, the horizontal SVE relates to the production goal through the destination approximation constraint (deviations from track that are larger than $\Delta \chi=90^{\circ}$ move the aircraft away from its destination).

The reduced forbidden area relates to the safety goal by showing the affordance of conflict, (the own velocity vector inside a reduced forbidden area indicates a conflict). Together with the internal maneuvering constraints from the SVE, it shows the affordance of avoidance: any vector within the SVE that is not inside any reduced forbidden area is a possible solution to a conflict. The reduced forbidden area also relates to the efficiency goal, through the 'shortest way out' principle [12]. The smallest vector change out of a reduced area will also result in the smallest path deviation. Note, however, that path deviation in the horizontal plane does not directly compare to path deviation in the vertical plane.

The forbidden area outlines link lower-level elements to higher level constraints. Together with the intruder symbols on the map displays, they link conflict and separation on the abstract function level to obstruction (motion), relative motion, and traffic location on the generalized and physical function levels. The tip of the triangle conveys intruder motion (the tip is offset from the center of the SVE by the intruder velocity vector), and the triangle bisector communicates intruder relative bearing, and therefore helps to link forbidden areas to their respective intruders.

\section{PRACTICAL APPLICATION}

An important argument in the current study is that in order to support operators in unforeseen situations, displays should go beyond visualizations that relate only to the automation logic. The interface should provide a window to the reasoning and functioning of the automation, by visualization of the affordances of the work domain, and by making clear how these affordances relate to the actions and advisories of the automation. The appropriateness of these displays for realworld applications, however, also depends on how well the concepts extend to complex situations, such as multiple intruder conflicts, complex trajectories, and of course situations where the automation is failing. This has also been considered for preceding concepts, and many of the properties illustrated in those studies apply to the current concept as well [12], [16].

Van Dam, for instance, illustrated that the forbidden areas work in a cumulative fashion [12]. Because each forbidden area reveals absolute maneuvering constraints, imposed by 
an intruder aircraft, a combination of forbidden areas from multiple aircraft, superimposed onto each other, will represent the set of states that would lead to a conflict with any one (or more) of these aircraft. As a result, any state outside of this combined constraint area is a solution to all of the current conflicts. This property is inherent to the presentation of constraints in an absolute velocity space.

Ellerbroek illustrated that conflicts can be solved in sequence, when the priority of each conflict is visualized using color coding of each forbidden area [16]. The current concept uses single colors for each forbidden area, where the color corresponds to the time to loss of separation, given the current state of ownship and intruder. A possible improvement could make use of the fact that every point in a forbidden area corresponds to a certain state vector, which in turn corresponds to a certain time to loss of separation. This way, each point in a forbidden area can be colored individually. In addition to visualization of the priorities of conflicts given the current state, the interface can then also reveal the viability of possible intermediate solutions in complex traffic situations.

One of the issues with co-planar displays is that the operator has to combine information from two displays to obtain a complete mental picture of the situation. Although the projected forbidden areas already provide strong links between the different elements on the displays, a crowded airspace can still make it difficult to make these links, especially when workload is already high. One of the techniques Woods proposes to improve this, is to provide across-display perceptual landmarks [55]. A common color, for instance, can provide a perceptual link between items on different displays that belong together. The priority color coding already partly fulfills this function, but can be improved with a selection system, where the different display elements that correspond to one intruder are highlighted upon selection.

The current concept employs the current states to derive the constraints imposed by other traffic. This method holds under the assumption that ownship and intruder state remain constant in the near future. When this is not the case, the affordance space will change as a function of space and time due to Trajectory Change Points (TCP), and other changes of state or intent. Several studies have illustrated methods to visualize intent in the forbidden areas [49]-[51]. Each of these methods makes use of the fact that the dimension along the bisector of the triangular forbidden area is related to the time at which the closest point of approach with the respective intruder will occur, with the triangle origin representing $t_{C P A}=\infty$. A change in state at $t=t_{T C P}$ will therefore result in a change in the forbidden area at the point where $t_{C P A}=t_{T C P}$. A similar method can be used to include intent in the current concept, by extending one of these methods to three dimensions.

Although current ATM concepts for unmanaged airspace suggest a traffic display to be used as a situation awareness tool for automated self-separation systems, constraint-based displays are not limited to this level of support. Because the displays visualize work-domain constraints and relations, they support the pilot on multiple levels of control, from fully automated conflict resolution, to manual pilot decision making.

\section{DISCUSSION}

The work presented in this paper is part of an ongoing study on the design of a trajectory planning aid. The goal is to obtain a graphical interface that supports pilots in their new task of airborne reconfiguration of a pre-planned trajectory, in case of traffic conflicts in unmanaged airspace. The current work focuses on ways to visualize 3-D data on a 2-D display. A co-planar display concept has been proposed, that is based on the previous top-down and side-view display concepts.

There are several reasons why a combination of a Horizontal Situation Display (HSD) and a Vertical Situation Display (VSD) was chosen for the co-planar display concept. First, these two displays provide the most intuitive maneuver space projections, and support the most straightforward resolution strategies, such as single-axis maneuvers, and combinations of speed and heading or vertical speed. A practical factor is also that these displays are already available on a modern flight deck, and they correspond closest to current re-planning tasks.

In previous research, the visualization of constraints on the display implicitly assumed that conflict geometries were flat: the constraints shown on the horizontal interface assume zero vertical separation and no vertical maneuvering, and the sideview display assumes that there is no cross track separation with intruder aircraft. When these assumptions are violated these displays will present overly conservative constraints. Simply combining these displays, therefore, is not sufficient to create an effective co-planar solution. Aside from the fact that good visual momentum demands visual cues that link both displays together, each individual display also requires modifications so that the presented constraints remain valid when a conflict can no longer be defined in just one plane. A co-planar display should reveal how individual planes interact with each other, and provide pilots with a consistent and intuitive view on what can be a complex, 3-D traffic situation.

The current concept, therefore, re-implements as much as possible the strong points of the previous, single-plane displays. The triangular shapes that result from planar projection of the 3-D traffic constraints provide strong and intuitive cues about the conflict geometry, reveal how different elements on the display belong together, and can help pilots keep an overview in complex traffic situations with multiple intruders. These projections are complemented with precisely derived constraints, that are also valid in combined cross-track and off-level conflict situations.

Although full, simultaneous 3-D maneuvering is still not consistently supported, it can be argued that this is a minor sacrifice when choosing a co-planar display over a perspective display: Several studies indicate that pilots prefer single-axis maneuvers [26], [33]-[36]. Also, the benefits (e.g., in terms of efficiency) of three-axis maneuvers over two- or one-axis maneuvers are rarely ever significant.

A possible downside of the constraint-based presentation in this concept is that in a densely populated airspace, the statevector envelope can become crowded with forbidden areas, making it less suitable (or unsuitable) as a situation awareness tool. Although this is an undesired situation, a de-cluttering algorithm will increase automation complexity, and reduce 
transparency of actions towards the operator. This will be the topic of a future study.

Current ATM concepts for the future of the structure of the airspace suggest a highly optimized, and -in certain parts of the airspace- decentralized system, with a high degree of automation. In the decentralized parts of airspace, aircraft will fly optimized, predetermined trajectories, where automation will be used to resolve problems that result from uncertainties during the flight. The concepts suggest that a display of traffic information should be added to help the aircrew judge these kinds of situations, and solve problems when they arise. The current study uses a constraint-based approach to design an interface that supports traffic situation awareness.

When used in combination with an automated system that provides explicit resolutions, such a display should improve operator trust and understanding of an automated resolution, by helping him understand how constraints shape possible resolutions. Note that this visualization is independent of the specific implementation of conflict resolution automation. Instead, it visualizes work domain information, which invariably forms the premise on which both automation and the human operator should base their actions. This method of visualization also provides an opportunity to create a visualization that is consistent across different levels of automation.

This method of display design, however, also implies that there are certain demands on the design of the automation. The interaction between automation and the human actor requires transparent functioning of the automated system. When a resolution advisory cannot consistently be explained by the information on a display, for instance because it takes additional (hidden) constraints into account, a pilot can hardly be asked to judge the fidelity of this resolution. Consistency between interface and automation, therefore, requires a common model of the work domain, from which the automation derives a resolution, and which the interface visualizes to the operator. This consistency will be crucial for human actors to share their cognition and decision-making with the automation.

\section{CONCLUSIONS}

A separation assistance display was proposed, that presents constraints on horizontal and vertical maneuvering, in a velocity action space that is overlaid on both a horizontal and a vertical situation display. A 2-D co-planar presentation was chosen because it offers an intuitive, undistorted and precise view on the constraints and the traffic situation. It also corresponds more closely to current-day flight-deck interfaces, as well as to pilot resolution preferences.

A follow-up paper (this issue) will present a set of experiments that evaluate this concept in terms of safety, performance, and situation awareness, in manual conflict resolution tasks. Future design iterations will focus on display clutter, intent, and on the interaction with different automated resolution modes.

\section{REFERENCES}

[1] Radio Technical Commission for Aeronautics, "Airborne Conflict Management: Application Description V2.5," Federal Aviation Authorities, Tech. Rep. RTCA SC-186, 2002.
[2] SESAR Consortium, "SESAR Definition Phase D3: The ATM Target Concept,' Eurocontrol, Tech. Rep. DLM-0612-001-02-00, 2007.

[3] D. A. Norman, "The "Problem" of Automation: Inappropriate Feedback and Interaction, not "Over-Automation"," Philosophical Transactions of the Royal Society of London, vol. 327, no. 1241, pp. 585-593, Apr. 1990.

[4] N. B. Sarter and D. D. Woods, "Pilot Interaction With Cockpit Automation: Operational Experiences With the Flight Management System," The International Journal of Aviation Psychology, vol. 2, no. 4, pp. 303-321, 1992.

[5] G. Lintern, T. Waite, and D. A. Talleur, "Functional Interface Design for the Modern Aircraft Cockpit," The International Journal of Aviation Psychology, vol. 9, no. 3, pp. 225-240, 1999.

[6] A. Q. V. Dao, S. Brandt, V. Battiste, K. P. Vu, T. Strybel, and W. W. Johnson, "The Impact of Automation Assisted Aircraft Separation on Situation Awareness," in Human Interface and the Management of Information. Information and Interaction. Springer, 2009, pp. 738747.

[7] K. J. Vicente, "Ecological Interface Design: Progress and Challenges," Human Factors, vol. 44, pp. 62-78, 2002.

[8] G. A. Jamieson, "Ecological Interface Design for Petrochemical Process Control: An Empirical Assessment," IEEE Transactions on Systems, Man, and Cybernetics, part A: Systems and Humans, vol. 37, no. 6, pp. 906-920, 2007.

[9] N. Dinadis and K. J. Vicente, "Designing Functional Visualizations for Aircraft System Status Displays," The International Journal of Aviation Psychology, vol. 9, no. 3, pp. 241-269, 1999.

[10] C. Borst, H. C. H. Suijkerbuijk, M. Mulder, and M. M. van Paassen, "Ecological Interface Design for Terrain Awareness," International Journal of Aviation Psychology, vol. 16, no. 4, pp. 375-400, 2006.

[11] C. Borst, F. A. Sjer, M. Mulder, M. M. van Paassen, and J. A. Mulder, "Ecological Approach to Support Pilot Terrain Awareness After Total Engine Failure," Journal of Aircraft, vol. 45, no. 1, pp. 159-171, 2008.

[12] S. B. J. van Dam, M. Mulder, and M. M. van Paassen, "Ecological Interface Design of a Tactical Airborne Separation Assistance Tool," IEEE Transactions on Systems, Man, and Cybernetics, part A: Systems and Humans, vol. 38, no. 6, pp. 1221-1233, 2008.

[13] F. M. Heylen, S. B. J. van Dam, M. Mulder, and M. M. van Paassen, "Design and Evaluation of a Vertical Separation Assistance Display," in AIAA Guidance, Navigation, and Control Conference and Exhibit, Honolulu (HI), 2008.

[14] A. van der Eijk, C. Borst, A. C. In 't Veld, M. M. van Paassen, and M. Mulder, "Assisting Air Traffic Control in Planning and Monitoring Continuous Descent Approach Procedures," Journal of Aircraft, vol. 49, no. 5, pp. 1376-1390, 2012.

[15] C. Borst, M. Mulder, and M. M. van Paassen, "Design and Simulator Evaluation of an Ecological Synthetic Vision Display," Journal of Guidance, Control and Dynamics, vol. 33, no. 5, pp. 1577-1591, 2010.

[16] J. Ellerbroek, M. Visser, S. B. J. van Dam, M. Mulder, and M. M. van Paassen, "Design of an Airborne Three-Dimensional Separation Assistance Display," IEEE Transactions on Systems, Man, and Cybernetics, part A: Systems and Humans, vol. 41, no. 6, pp. 863-875, 2011.

[17] K. J. Vicente and J. Rasmussen, "Ecological Interface Design: Theoretical Foundations," IEEE Transactions on Systems, Man, and Cybernetics, vol. 22, no. 4, pp. 589-606, 1992.

[18] C. M. Burns and J. R. Hajdukiewicz, Ecological Interface Design. FL: Boca Raton: CRC Press LLC, 2004.

[19] J. J. Gibson, "The Theory of Affordances," Perceiving, Acting and Knowing: Toward an Ecological Psychology, pp. 67-82, 1977.

[20] - The Ecological Approach to Visual Perception. Houghton Mifflin, 1979.

[21] K. Christoffersen, C. N. Hunter, and K. J. Vicente, "A Longitudinal Study of the Effects of Ecological Interface Design on Deep Knowledge," International Journal of Human-Computer Studies, vol. 48, pp. 729-762, 1998.

[22] E. J. Bass and A. R. Pritchett, "Human-Automated Judge Learning: A Methodology for Examining Human Interaction With Information Analysis Automation," IEEE Transactions on Systems, Man, and Cybernetics, part A: Systems and Humans, vol. 38, no. 4, pp. 759-776, 2008.

[23] M. S. T. Carpendale, D. J. Cowperthwaite, and F. D. Fracchia, "Extending Distortion Viewing from 2D to 3D," Computer Graphics and Applications, IEEE, vol. 17, no. 4, pp. 42-51, 1997.

[24] M. St John, M. B. Cowen, H. S. Smallman, and H. M. Oonk, "The Use of 2D and 3D Displays for Shape-Understanding versus RelativePosition Tasks," Human Factors, vol. 43, no. 1, pp. 79-98, 2001. 
[25] S. R. Ellis, M. W. McGreevy, and R. J. Hitchcock, "Perspective Traffic Display Format and Airline Pilot Traffic Avoidance," Human Factors, vol. 29, no. 4, pp. 371-382, 1987.

[26] A. L. Alexander, C. D. Wickens, and D. H. Merwin, "Perspective and Coplanar Cockpit Displays of Traffic Information: Implications for Maneuver Choice, Flight Safety, and Mental Workload," The International Journal of Aviation Psychology, vol. 15, pp. 1-21, 2005.

[27] L. C. Thomas and C. D. Wickens, "Display Dimensionality, Conflict Geometry, and Time Pressure Effects on Conflict Detection and Resolution Performance Using Cockpit Displays of Traffic Information," The International Journal of Aviation Psychology, vol. 16, no. 3, pp. 321-342, 2006.

[28] S. N. Roscoe, "Airborne Displays for Flight and Navigation," Human Factors, vol. 10, no. 4, pp. 321-332, 1968.

[29] C. D. Wickens, "The When and How of Using 2-D and 3-D Displays for Operational Tasks," in Proceedings of the Human Factors and Ergonomics Society, 2000, pp. 403-406.

[30] M. L. Bolton, E. J. Bass, and J. R. Comstock, "Spatial Awareness in Synthetic Vision Systems: Using Spatial and Temporal Judgments to Evaluate Texture and Field of View," pp. 961-974, 2007.

[31] M. W. McGreevy and S. R. Ellis, "The Effect of Perspective Geometry on Judged Direction in Spatial Information Instruments," Human Factors, vol. 28, no. 4, pp. 439-456, 1986

[32] M. L. Bolton and E. J. Bass, "Using Relative Position and Temporal Judgments to Identify Biases in Spatial Awareness for Synthetic Vision Systems," The International Journal of Aviation Psychology, vol. 18 no. 2, pp. 183-206, 2008.

[33] J. M. Hoekstra, "Designing for Safety: The Free Flight Air Traffic Management Concept," Ph.D. dissertation, Delft University of Technology, The Netherlands, 2001.

[34] C. D. Wickens, J. Helleberg, and X. Xu, "Pilot Maneuver Choice and Workload in Free Flight," Human Factors and Ergonomics Society Annual Meeting Proceedings, vol. 44, no. 2, pp. 171-188, 2002.

[35] C. L. A. Steens, S. B. J. van Dam, M. M. van Paassen, and M. Mulder, "Comparing Situation Awareness for Two Airborne Separation Assistance Interfaces," in AIAA Guidance, Navigation and Control Conference and Exhibit, Honolulu (HI), 2008.

[36] J. Ellerbroek, M. M. van Paassen, and M. Mulder, "Evaluation of a Separation Assistance Display in a Multi-Actor Experiment," IEEE Transactions on Human-Machine Systems, submitted, 2011.

[37] J. K. Kuchar and L. C. Yang, "A Review of Conflict Detection and Resolution Modelling Methods," IEEE Transactions on Intelligent Transportation Systems, vol. 1, no. 4, pp. 179-189, 2000.

[38] R. Ghosh and C. J. Tomlin, "Maneuver Design for Multiple Aircraft Conflict Resolution," in Proceedings of the American Control Conference, Chicago (IL), 2000, pp. 672-676.

[39] J. M. Hoekstra, R. N. H. W. van Gent, and R. C. J. Ruigrok, "Designing for Safety: the Free Flight Air Traffic Management Concept," Reliability Engineering and System Safety, vol. 75, pp. 215-232, 2002.

[40] L. Pallottino, E. M. Feron, and A. Bicchi, "Conflict Resolution Problems for Air Traffic Management Systems Solved With Mixed Integer Programming," IEEE Transactions on Intelligent Transportation Systems, vol. 3, no. 1, pp. 3-11, Mar. 2002.

[41] D. J. Wing, R. A. Vivona, and D. A. Roscoe, "Airborne Tactical IntentBased Conflict Resolution Capability," in 9th AIAA Aviation, Technology, Integration, and Operations, 2009.

[42] C. Meckiff and P. Gibbs, "PHARE Highly Interactive Problem Solver," Eurocontrol, Tech. Rep. 273/94, Nov. 1994.

[43] V. Battiste, W. W. Johnson, N. H. Johnson, S. Granada, and A. Q. V. Dao, "Flight Crew Perspective on the Display of 4D Information for En Route and Arrival Merging and Spacing," Human-Computer Interaction: Interaction Platforms and Techniques, pp. 541-550, 2007.

[44] W. R. Knecht, "Testing a Multidimensional Nonveridical Aircraft Collision Avoidance System," Human Factors, vol. 50, no. 4, pp. 565-575, 2008.

[45] E. R. Tufte, Envisioning Information. Cheshire, CT: Graphics Press, 1990.

[46] S. K. Ojha, Flight Performance of Aircraft. AIAA Education Series, 1995.

[47] Radio Technical Commission for Aeronautics, "Final Report of the RTCA Board of Directors' Select Committee on Free Flight," RTCA, Tech. Rep., 1995.

[48] Federal Aviation Administration and Eurocontrol, "Principles of Operation for the Use of Airborne Separation Assurance Systems," Federal Aviation Authorities - Eurocontrol, Tech. Rep. PO-ASAS-V7.1, 2001.
[49] S. B. J. van Dam, M. Mulder, and M. M. van Paassen, "The Use of Intent Information in an Airborne Self-Separation Assistance Display Design," in AIAA Guidance, Navigation, and Control Conference and Exhibit, 2009.

[50] J. D'Engelbronner, M. Mulder, M. M. van Paassen, S. de Stigter, and H. Huisman, "The Use of the Dynamic Solution Space to Assess Air Traffic Controller Workload," in AIAA Guidance, Navigation, and Control Conference and Exhibit, 2010.

[51] G. A. Mercado-Velasco, M. Mulder, and M. M. van Paassen, "Analysis of Air Traffic Controller Workload Reduction Based on the Solution Space for the Merging Task," in AIAA Guidance, Navigation, and Control Conference and Exhibit, 2010.

[52] R. A. Paielli, "Modeling Maneuver Dynamics in Air Traffic Conflict Resolution," Journal of Guidance Control and Dynamics, vol. 26, no. 3 , pp. 407-415, May 2003.

[53] S. B. J. van Dam, M. Mulder, and M. M. van Paassen, "Airborne Self-Separation Display with Turn Dynamics and Intruder IntentInformation," in IEEE International Conference on Systems, Man and Cybernetics. Montreal, Canada: IEEE, 2007.

[54] U.S. Department of Transportation and Federal Aviation Administration, "Introduction to TCAS II Version 7.1," 2011.

[55] D. D. Woods, "Visual Momentum: a Concept to Improve the Cognitive Coupling of Person and Computer," International Journal of HumanComputer Studies, vol. 21, pp. 229-244, 1984.

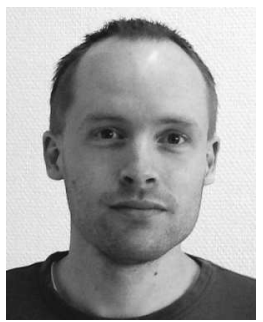

Joost Ellerbroek received the M.Sc. degree in aerospace engineering from the Delft University of Technology, The Netherlands, in 2007, where he is currently working toward the Ph.D. degree. His $\mathrm{Ph} . \mathrm{D}$. work concentrates on the design and validation of an interface that supports interaction with airborne separation automation. The research presented in this paper is part of his thesis.

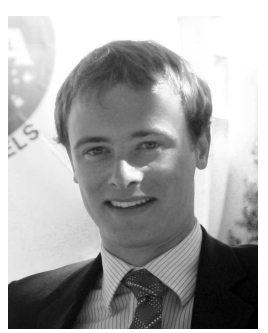

Koen C. R. Brantegem received the M.Sc. degree (cum laude) from the Delft University of Technology, The Netherlands, in 2011. He graduated within the control and simulation section on his thesis entitled "Ecological 2-D Coplanar Airborne Separation Assurance System". The results of his work are incorporated in this paper. He is currently working towards obtaining a commercial pilot license.

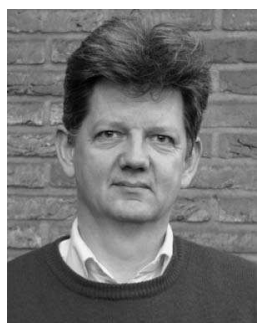

M. M. (René) van Paassen received the M.Sc. degree (1988, cum laude) from the Delft University of Technology, The Netherlands, and a Ph.D. (1994), on the neuromuscular system of the pilot's arm. He thereafter was a Brite/EuRam Research Fellow with the University of Kassel, and a post-doc at the Technical University of Denmark. Currently, he is associate professor at the faculty of Aerospace Engineering, Delft University of Technology. His work ranges from studies of perceptual processes and manual control to complex cognitive systems. 


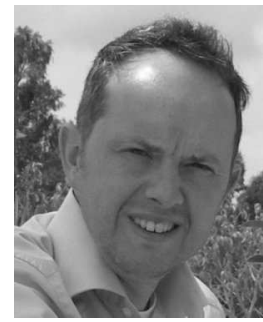

Max Mulder received the M.Sc. (1992) and Ph.D. degrees (1999, cum laude) from the Delft University of Technology, The Netherlands, for his work on the cybernetics of tunnel-in-the-sky displays. He is currently Full Professor and Head of the Control and Simulation Section, Faculty of Aerospace Engineering, Delft University of Technology. His research interests include cybernetics and its use in modeling human perception and performance, and cognitive systems engineering and its application in the design of "ecological" human-machine interfaces. 